\title{
A COMPARISON OF DECISION TREE-BASED MODELS FOR FOREST ABOVE- GROUND BIOMASS ESTIMATION USING A COMBINATION OF AIRBORNE LIDAR AND LANDSAT DATA
}

\author{
Haifa Tamiminia ${ }^{\text {a, *, }}$ Bahram Salehi ${ }^{\text {a }}$, Masoud Mahdianpari ${ }^{\mathrm{b}}$, Colin M. Beier ${ }^{\mathrm{c}}$, Lucas Johnson ${ }^{\mathrm{c}}$, and Daniel B. Phoenix ${ }^{\mathrm{c}}$
}

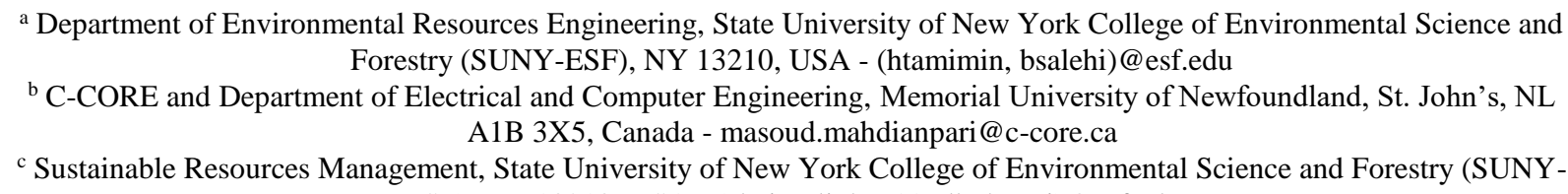
ESF), NY 13210, USA - (cbeier, ljohns11, dbphoeni)@esf.edu

Commission III, WG III/10

KEY WORDS: Above-ground Biomass, Decision Tree-based Models, Deep Forest, Random Forest, Decision Tree, Remote Sensing, Machine Learning.

\begin{abstract}
:
Forest is one of the most crucial Earth's resources. Forest above-ground biomass (AGB) mapping has been research endeavors for a long time in many applications since it provides valuable information for carbon cycle monitoring, deforestation, and forest degradation monitoring. A methodology to rapidly and accurately estimate AGB is essential for forest monitoring purposes. Thus, the main objective of this paper was to investigate the performance of decision tree-based models to predict AGB at a site in Huntington Wild Forest (HWF) in Essex County, NY using continuous forest inventory (CFI) plots. The results of decision tree, random forest, and deep forest regression models were compared using light detection and ranging (LiDAR), Landsat 5 $\mathrm{TM}$, and a combination of them. The results illustrated the importance of integration of Landsat $5 \mathrm{TM}$ and LiDAR data, which benefits from both vertical forest structure and spectral information reflected by canopy cover. In addition, the deep forest model with a root mean square error (RMSE) of $51.63 \mathrm{Mg} / \mathrm{ha}$ and $\mathrm{R}$-squared $\left(\mathrm{R}^{2}\right)$ of 0.45 outperformed other regression tree-based models, regardless of the dataset.
\end{abstract}

\section{INTRODUCTION}

Forest is considered as one of the most valuable Earth resources, which is required to be monitored in a timely manner (Bastin et al. 2017). Sustainable forest management is of paramount significance for many applications, namely forest productivity, monitoring carbon sequestration, and investigating deforestation. Importantly, forest aboveground biomass (AGB) plays a crucial role in carbon sequestration, which contributes to global climate change issues (M. Li, Im, and Beier 2013). Accurate AGB estimation has been an area of interest for many researchers. Conventional field measurement techniques provide an accurate estimation of AGB while they are labor-intensive, costly, time-consuming, and not applicable for large regions (M. Li, Im, and Beier 2013). In recent years, remote sensing data paved the road for a cost-effective AGB estimation over large areas.

Optical and synthetic aperture radar (SAR) imagery are valuable sources for forest monitoring applications. However, saturation is the most common issue with these datasets (Joshi et al. 2017; Kachamba et al. 2016). It worth mentioning that saturation occurs in forests with multilayer canopies or high dense biomass when spectral reflectance values of pixels are not sensitive to biomass changes, which affects the quality of AGB estimation (Zhao et al. 2016). Zhao et al. (2016) reported that saturation is more severe for AGB values greater than $130 \mathrm{Mg} / \mathrm{ha}$. In addition, weather conditions (e.g., rain, snow, shadow, and cloud cover) can greatly affect the quality of the optical data. Light detection and ranging (LiDAR) is another remote sensing data, which directly measures the vertical structure of forest canopy (Boudreau et al. 2008). Although LiDAR can provide valuable information for AGB estimation, it is costly and limited for large-scale applications.

So far, many studies have been concentrated on leveraging remote sensing data for AGB estimation (Issa et al. 2020; Dube et al. 2016). These studies have compared different remote sensing datasets and reported the achieved results. Several studies have been focusing on combining the LiDAR, optical, and SAR data to maximize the potential of these datasets for AGB estimation (Shao, Zhang, and Wang 2017; Urbazaev et al. 2018). Zhang et al. (2019) and Cao et al. (2018) used the integration of LiDAR and optical imagery to improve the estimation of AGB. The combination of SAR data with optical and LiDAR data has been used by Shao and Zhang (2016) and Hyde et al. (2007) which enhanced AGB prediction results.

Machine learning techniques are one of the commonly used models in AGB estimation since it is more compatible with the non-linear inherent characteristic of remote sensing data (C. Li, Li, and $\mathrm{Li}$ 2020). Among machine learning algorithms, decision tree-based models have shown better performance in AGB prediction (Y. Li et al. 2020). The

\footnotetext{
* Corresponding Author
} 
random forest $(\mathrm{RF})$ regression algorithm has been widely used in AGB estimation and has shown promising results (Mutanga, Adam, and Cho 2012; Dang et al. 2019; Karlson et al. 2015).

According to the existing papers on forest AGB estimation using remote sensing data, there is always room to fully explore the potential of models and datasets to improve the accuracy of AGB estimation. The main objective of this paper is to address the capability of the combination of LiDAR and optical data for accurate AGB estimation. In order to achieve this goal, three well-known decision treebased machine learning algorithms are implemented using the integration of Landsat 5 thematic mapper (TM) imagery and airborne LiDAR data. Thus, this study presents a comprehensive comparison between decision tree (DT), RF, and deep forest regression models.

This study focuses on investing the following research aims: 1) assessing the potential of integration of Landsat 5 TM and LiDAR data for AGB estimation, 2) comparing decision tree-based algorithms for predicting AGB values, 3) evaluating whether deep forest model can provide better results in comparison to DT and RF.

\section{STUDY AREA AND DATA}

\subsection{Study Area}

This project was conducted on the Huntington Wildlife Forest (HWF) area, which is located in the central Adirondack Park in northern New York State (Figure 1). HWF, with an approximate area of 6,000 ha (latitude 44E $00 " \mathrm{~N}$, longitude 74E 13" W), was donated to the State University of New York, College of Environmental Science and Forestry (SUNY-ESF) for research purposes. The elevation of the mountainous topography of HWF property ranges from $473 \mathrm{~m}$ to $908 \mathrm{~m}$ above mean sea level. Huntington has a mean annual temperature of 4.4 Celsius degree and a mean annual precipitation of $1010 \mathrm{~mm}(\mathrm{~S}$. Li, Quackenbush, and Im 2019). Huntington forest contains $72 \%$ of northern hardwoods, $18 \%$ of mixed hardwood conifer, and $10 \%$ of conifer species.

\subsection{Field Inventory Data}

In this study, SUNY-ESF continuous forest inventory (CFI) plots have been used as reference data. This comprehensive dataset was collected during the summer of 2011. The CFI dataset of HWF in 2011 contained 288 sample plots with approximately $807 \mathrm{~m}^{2}$ circular regions. In each sample plot, all trees with a diameter at breast height $(\mathrm{DBH})$ of $11.7 \mathrm{~cm}$ and greater were measured. For each tree, tree species, $\mathrm{DBH}$, and the relative location to the center of the sample plot were recorded (S. Li, Quackenbush, and Im 2019). Then, AGB at the tree level was calculated using speciesspecific DBH allometric equations (Kennedy et al. 2018). Finally, plot-level AGB was calculated as the average AGB per unit area within each sample plot (S. Li, Quackenbush, and Im 2019). In other words, the plot level AGB in megagrams per hectare $(\mathrm{Mg} / \mathrm{ha})$ was calculated by dividing the tree level AGB by the plot area.

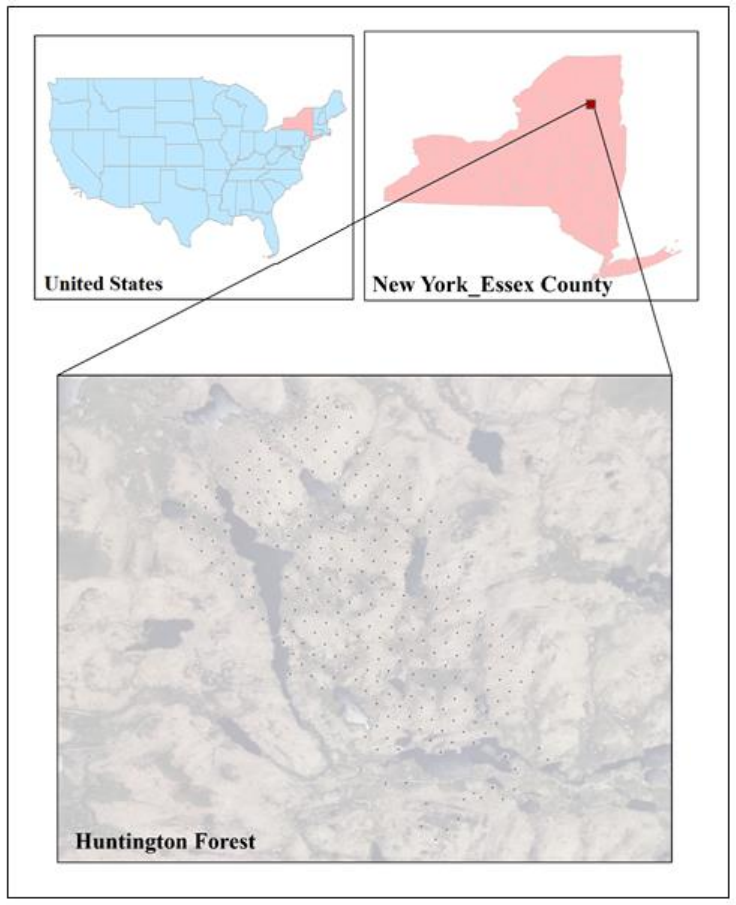

Figure 1. Location of the study area (Huntington Wildlife Forest) in Essex County, NY for forest AGB estimation using decision-tree based models. Black circles indicate sample plots located in Huntington wildlife forest.

\subsection{LiDAR data}

Discrete return LiDAR data collection was acquired over HWF in May 2015 using the Leica Airborne Laser Scanner (ALS70). First, a k-nearest neighbor imputation algorithm $(\mathrm{k}=5)$ was used to convert the raw point clouds into heightnormalized point clouds. Then, predictors were computed using the height normalized LiDAR data for modeling at 30 $\mathrm{m}$ grid cells. Finally, 29 predictors were computed and fed as inputs into the machine learning models (Table 1). Since field measurements were collected in 2011, the main hypothesis was that HWF did not change from 2011 to 2015.

\subsection{Landsat 5 TM Imagery}

Google Earth Engine (GEE) cloud platform was used to process and download the Landsat 5 imagery, and then $\mathrm{R}$ software was used to train the model and estimate AGB values. Spectral bands and some biomass-related vegetation indices were used to train the regression models. Table 2 lists vegetation indices used in this study. Spectral bands were extracted using Landsat 5 TM imagery in 2011 for HWF. Landsat 5 dataset contains three visible, one nearinfrared (NIR) band, and two short-wave infrared (SWIR) bands with $30 \mathrm{~m}$ resolution. These images are atmospherically ortho-corrected surface reflectance. A cloud mask was applied to the imagery to remove the cloud effect in the acquired images. 


\begin{tabular}{|c|c|c|c|}
\hline Predictor & Description & Predictor & Description \\
\hline pgi & $\begin{array}{l}\text { Percentage of } \\
\text { ground } \\
\text { intensity }\end{array}$ & z_kurt & $\begin{array}{c}\text { Kurtosis of } \\
\text { height }\end{array}$ \\
\hline pfi & $\begin{array}{c}\text { Percentage of } \\
\text { feature } \\
\text { intensity }\end{array}$ & z_skew & $\begin{array}{c}\text { Skewness of } \\
\text { height }\end{array}$ \\
\hline cv_i & $\begin{array}{l}\text { Coefficient of } \\
\text { variation of } \\
\text { intensity }\end{array}$ & L3 & 3rd L-moment \\
\hline skew_i & $\begin{array}{l}\text { Skewness of } \\
\text { intensity }\end{array}$ & L_cv & $\begin{array}{c}\text { L-moment } \\
\text { coefficient of } \\
\text { variation }\end{array}$ \\
\hline kurt_i & $\begin{array}{c}\text { Kurtosis of } \\
\text { intensity }\end{array}$ & L_kurt & $\begin{array}{c}\text { L-moment } \\
\text { kurtosis }\end{array}$ \\
\hline piz25 & $\begin{array}{l}\text { Percentage of } \\
\text { intensity } \\
\text { above the } \\
\text { 25th height } \\
\text { percentile }\end{array}$ & h10 & $\begin{array}{l}\text { 10th height } \\
\text { percentile }\end{array}$ \\
\hline piz50 & $\begin{array}{l}\text { Percentage of } \\
\text { intensity } \\
\text { above the } \\
\text { 50th height } \\
\text { percentile }\end{array}$ & h20 & $\begin{array}{l}\text { 20th height } \\
\text { percentile }\end{array}$ \\
\hline piz75 & $\begin{array}{l}\text { Percentage of } \\
\text { intensity } \\
\text { above the } \\
\text { 75th height } \\
\text { percentile }\end{array}$ & h40 & $\begin{array}{l}\text { 40th height } \\
\text { percentile }\end{array}$ \\
\hline piz90 & $\begin{array}{l}\text { Percentage of } \\
\text { intensity } \\
\text { above the } \\
\text { 90th height } \\
\text { percentile }\end{array}$ & h70 & $\begin{array}{l}\text { 70th height } \\
\text { percentile }\end{array}$ \\
\hline piz99 & $\begin{array}{l}\text { Percentage of } \\
\text { intensity } \\
\text { above the } \\
\text { 99th height } \\
\text { percentile }\end{array}$ & h80 & $\begin{array}{l}\text { 80th height } \\
\text { percentile }\end{array}$ \\
\hline $\mathrm{cv}$ & $\begin{array}{c}\text { Coefficient of } \\
\text { variation of } \\
\text { height }\end{array}$ & h90 & $\begin{array}{l}\text { 90th height } \\
\text { percentile }\end{array}$ \\
\hline $\mathrm{d} 20$ & $\begin{array}{l}\text { Percent of } \\
\text { returns above } \\
\text { the } 2 \text { nd height } \\
\text { bin (of } 10 \\
\text { equal bins) }\end{array}$ & h95 & $\begin{array}{l}\text { 95th height } \\
\text { percentile }\end{array}$ \\
\hline $\mathrm{d} 40$ & $\begin{array}{l}\text { Percent of } \\
\text { returns above } \\
\text { the } 4 \text { th height } \\
\text { bin (of } 10 \\
\text { equal bins) }\end{array}$ & h99 & $\begin{array}{l}\text { 99th height } \\
\text { percentile }\end{array}$ \\
\hline d60 & $\begin{array}{l}\text { Percent of } \\
\text { returns above } \\
\text { the } 6 \text { th height } \\
\text { bin (of } 10 \\
\text { equal bins) }\end{array}$ & $\mathrm{d} 70$ & $\begin{array}{l}\text { Percent of } \\
\text { returns above } \\
\text { the } 7 \text { th height } \\
\text { bin (of } 10 \\
\text { equal bins) }\end{array}$ \\
\hline d90 & $\begin{array}{l}\text { Percent of retu } \\
10 \text { equal bins) }\end{array}$ & bove the & h height bin (of \\
\hline
\end{tabular}

Table 1. LiDAR predictors used to estimate AGB over Huntington Wildlife Forest.

\begin{tabular}{|c|c|}
\hline Vegetation Indices & Formula \\
\hline \multirow{2}{*}{$\begin{array}{c}\text { Normalized Difference } \\
\text { Vegetation Index (NDVI) }\end{array}$} & NIR-R \\
\hline & $\overline{\mathrm{NIR}+\mathrm{R}}$ \\
\hline \multirow{2}{*}{$\begin{array}{l}\text { Ratio Vegetation Index } \\
\text { (RVI) }\end{array}$} & NIR \\
\hline & $\mathrm{R}$ \\
\hline $\begin{array}{c}\text { Difference Vegetation } \\
\text { Index(DVI) }\end{array}$ & NIR-R \\
\hline $\begin{array}{l}\text { Soil Adjusted Vegetation } \\
\text { Index (SAVI) }\end{array}$ & $\frac{\mathrm{NIR}-\mathrm{R}}{\mathrm{NIR}+\mathrm{R}+\mathrm{L}}(1+\mathrm{L}), \mathrm{L}=0.5$ \\
\hline \multirow{2}{*}{$\begin{array}{c}\text { Normalized Green-Red } \\
\text { Difference Index (NGRDI) }\end{array}$} & G-R \\
\hline & $\overline{\mathrm{G}+\mathrm{R}}$ \\
\hline \multirow{2}{*}{$\begin{array}{c}\text { Wide Dynamic Range } \\
\text { Vegetation Index (WDRVI) }\end{array}$} & $0.1^{*}$ NIR-Red \\
\hline & $\overline{0.1 * \mathrm{NIR}+\mathrm{Red}}$ \\
\hline Excess Green Index (ExG) & 2G-Red-Blue \\
\hline $\begin{array}{l}\text { Chlorophyll Index-green (CI } \\
\text { green) }\end{array}$ & $\frac{\mathrm{NIR}}{\mathrm{G}}-1$ \\
\hline Visible Atmospherically & G-R \\
\hline Resistant Index (VARI) & $\overline{\mathrm{G}+\mathrm{R}-\mathrm{B}}$ \\
\hline $\begin{array}{l}\text { Chlorophyll Vegetation } \\
\text { Index (CVI) }\end{array}$ & $\mathrm{NIR} * \frac{\mathrm{R}}{\mathrm{G}^{2}}$ \\
\hline
\end{tabular}

Table 2. Vegetation indices derived from Landsat 5 TM imagery for AGB estimation over Huntington Wildlife Forest.

\section{METHODS}

In this paper, three decision tree-based machine learning regression models including DT, RF, and deep forest were deployed and compared. Decision tree-based algorithms are a subset of ensemble learning which help to decrease the variance and increase the stability (Dey 2016). The R software and Python 3.7 packages were used to implement regression models and predict AGB for LiDAR data, Landsat $5 \mathrm{TM}$ imagery, and integration of LiDAR and Landsat 5 TM data. Each model was run using a training/testing split of 70/30 to calculate the root mean square error (RMSE) and R-squared (R2). The following subsections describe a brief background and parameters regarding each model. Parameters were tuned through a grid search approach.

\subsection{Decision Tree (DT)}

DT is the most popular machine learning technique which builds the foundation of tree-based models (Kotsiantis 2013). It develops a regression model based on a tree structure of the conditional statement. DT uses attributes in the dataset to break down the data into smaller subsets by making decisions. Nodes can be divided into two categories: decision nodes and leaf nodes. The former specifies decisions to split the data while the latter defines the value of the attributes. DT provides straightforward interpretation and manages non-linear data. However, DT is prone to over-fitting, and a small noise in the input dataset can remarkably influence the predictions (Song and Ying 2015). Package "rpart" in R was used to implement the DT 
model. Figure 2 shows the selected parameters after the grid search approach for the combination of LiDAR and Landsat data. The complexity parameter (cp) is the minimum improvement needed at each node. The minsplit defines the minimum number of observations in the root node (decision node) that could be broken down. The minbucket denotes the smallest number of observations in a leaf node. The maxdepth prevents the tree growth from a certain depth.

\subsection{Random Forest}

Random forest is an ensemble non-parametric method, which combines many decision trees in parallel (Mahdianpari et al. 2017). It uses a combination of bagging, which randomly selects variables, but with replacement, as training for growing the tree. If there are $M$ input predictors, then $\mathrm{m} \leq \mathrm{M}$ predictors are selected randomly out of $\mathrm{M}$, and the best split on $\mathrm{m}$ is used to split the node. Each tree is grown to the largest possible extent without pruning (Ali et al. 2012). RF uses a bagging technique to make sure variety in trees, thus reduces the over-fitting. Moreover, it can handle noisy datasets. An $\mathrm{R}$ package named "randomForest" was used for RF model training. The list of selected parameters is shown in Figure 2. The ntree is the number of trees in a forest. The mtry defines the number of random variables at each split. The nodesize is the minimum number of samples within the leaf nodes.

\subsection{Deep Forest}

Deep forest is a novel decision tree ensemble approach, which can be considered as an alternative for deep neural networks (DNNs) with fewer hyper-parameters and complexity (Zhou and Feng 2017). In contrast to DNNs, deep forest runs faster, and it is much easier to train. While DNNs require large-scale training data, deep forest can perform well with small-scale training data (Zhou and Feng 2017). This approach is also known as multi-grained cascade forest (gcForest). A cascade structure enables deep forest to do representation learning, while in DNNs, representation learning is done by the layer-by-layer processing of features. In deep forest, each level of cascade gets the feature information processed by its preceding level and gives its processing output to the next level (Zhou and Feng 2017). The number of cascade levels can be adaptively determined to perform well even with smallscale data (Zhou and Feng 2017). It is worth mentioning that each level is an ensemble of random forests (e.g. an ensemble of ensembles). This method contains different types of forests to increase the diversity which is required for ensemble constructions. In order to implement the deep forest model, a combination of gcForest and sklearn packages in $\mathrm{R}$ and Python was utilized. Figure 2 shows the selected parameters for deep forest implementation. The $\mathrm{n}$ _cascadeRF defines the number of random forests in a cascade layer, while n_cascadeRFtree specifies the number of trees in a single random forest of a cascade layer. The n_mgsRFtree defines the number of trees in an RF during multi grain scanning.

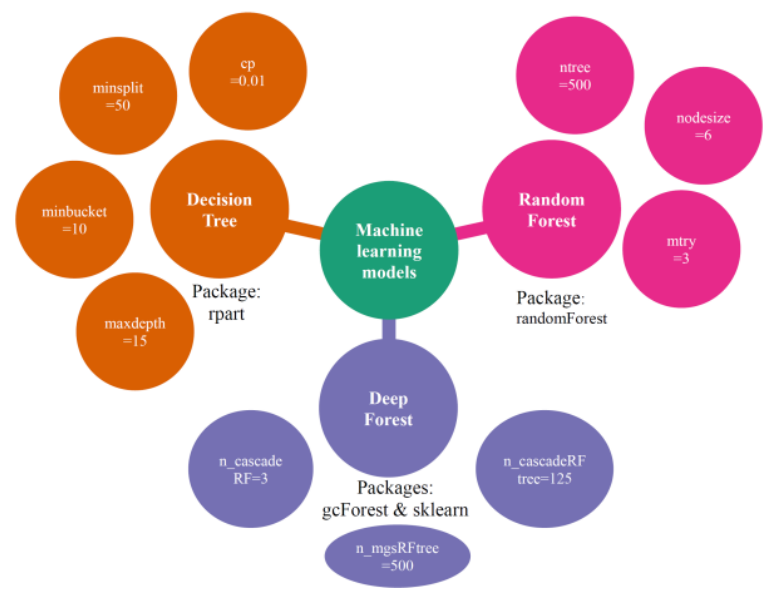

Figure 2. Packages and selected parameters used for the implementation of decision tree-based models for AGB estimation (Huntington Wildlife Forest) in Essex, NY using the combination of LiDAR and Landsat data.

\section{RESULTS AND DISCUSSION}

This section represents the results of implemented decision tree-based models on LiDAR, Landsat $5 \mathrm{TM}$, and a combination of LiDAR and Landsat 5 TM data. Table 3 summarizes the RMSE and $\mathrm{R}^{2}$ of DT, RF, and deep forest models. In addition, AGB maps produced by each regression model are demonstrated in Figure 3.

\begin{tabular}{ccccc}
\hline Model & & LiDAR & Landsat 5 & $\begin{array}{c}\text { LiDAR + } \\
\text { Landsat 5 }\end{array}$ \\
\hline $\begin{array}{c}\text { Decision } \\
\text { Tree }\end{array}$ & $\begin{array}{c}\mathrm{RMSE} \\
(\mathrm{Mg} / \mathrm{ha})\end{array}$ & 58.37 & 70.94 & 59.23 \\
\cline { 2 - 5 } $\mathrm{R}^{2}$ & 0.33 & 0.21 & 0.32 \\
\hline $\begin{array}{c}\mathrm{RMSE} \\
\text { Fandom }\end{array}$ & $\begin{array}{c}\mathrm{Mg} / \mathrm{ha}) \\
\mathrm{R}^{2}\end{array}$ & 53.51 & 67.11 & 52.67 \\
\hline $\begin{array}{c}\mathrm{RMSE} \\
\text { Deep }\end{array}$ & \begin{tabular}{c}
$\mathrm{Mg} / \mathrm{ha})$ \\
\cline { 2 - 5 }
\end{tabular} & 52.01 & 67.22 & 0.44 \\
\hline $\mathrm{R}^{2}$ & 0.43 & 0.23 & 0.45 \\
\hline
\end{tabular}

Table 3. Results of HWF AGB estimation using decision tree, random forest, and deep forest models and integration of LiDAR and Landsat 5 TM imagery.

As shown in Table 3, LiDAR data provides a smaller RMSE than Landsat data, which indicates the importance of vertical structure captured by LiDAR data. Although tree diameters are more related to AGB, height characteristics derived by LiDAR data can be efficiently used for AGB estimation (Zhao et al. 2016). The most probable issue with the low performance of Landsat imagery might be due to the saturation problem. The AGB of HWF varies from 0 to 433.2 Mg/ha (Table 4). Landsat $5 \mathrm{TM}$ suffers from saturation that greatly influences the RMSE and R2. Thus, in this study area, using Landsat-only imagery is not the best option for accurate AGB estimation. By combining LiDAR and Landsat 5 TM imagery, RMSE decreases, which is a great sign of improvement in AGB estimation. The reason behind this improvement is that using an 
integration of LiDAR and Landsat will benefit from both vertical and spectral information. Thus, the AGB estimation will improve. The trend of performance increasing by using both LiDAR and Landsat data can be seen in all three regression models.

\begin{tabular}{ccccc}
\hline $\begin{array}{c}\text { Min } \\
(\mathrm{Mg} / \mathrm{ha})\end{array}$ & $\begin{array}{c}1^{\text {st }} \\
\text { quartile } \\
(\mathrm{Mg} / \mathrm{ha})\end{array}$ & $\begin{array}{c}\text { Median } \\
(\mathrm{Mg} / \mathrm{ha})\end{array}$ & $\begin{array}{c}3^{\text {rd }} \text { quartile } \\
(\mathrm{Mg} / \mathrm{ha})\end{array}$ & $\begin{array}{c}\mathrm{Max} \\
(\mathrm{Mg} / \mathrm{ha})\end{array}$ \\
\hline 0 & 139.5 & 183.2 & 183.4 & 433.2 \\
\hline
\end{tabular}

Table 4. Statistical characteristics of AGB of CFI plots in HWF used for AGB estimation using decision tree-based models and remote sensing data.

The DT model provided the highest RMSE and the lowest $\mathrm{R}^{2}$ for all datasets except the combination of LiDAR and Landsat. DT model tends to over-fit which greatly affects its performance. Over-fitting occurs when the model fits very well to the training data, while for the testing data, it cannot predict the correct values since the model knows details about training data, including noise. The second-best model was RF, which provided better results in terms of RMSE and $\mathrm{R}^{2}$ for all datasets in comparison to DT model. The RF uses an ensemble of trees, which reduces the overfitting issues (Mahdianpari et al. 2017). In addition, it can overcome the noise in the dataset. Deep forest provided slightly better results for Landsat and a combination of LiDAR and Landsat datasets. The best improvement of deep forest can be seen in LiDAR dataset. Recently, deep forests are considered the best alternative for DNNs; still, their potentials need to be investigated. In this study area, deep forest with its own unique characteristics, such as layer-by-layer processing, sufficient model complexity, and faster training than DNNs (Zhou and Feng 2017), could more accurately estimate the AGB in comparison to DT and RF.

Figure 3 shows the AGB maps produced using the combination of Landsat $5 \mathrm{TM}$ and LiDAR data, which provided the best results in terms of RMSE and $\mathrm{R}^{2}$ for all regression tree models. The maximum range of AGB is limited to $350 \mathrm{Mg} / \mathrm{ha}$ since there is no estimated AGB above this range due to the saturation issue with high biomass. As seen in Figure 3, both deep forest and RF were capable of predicting AGB within a wider range than DT. DT did a poor job in estimating biomass with low and high values. Furthermore, the histograms of the three AGB maps are plotted to provide more information about the raster (Figure 4). All three maps suffer from saturation issues, and they cannot estimate AGB values for more than $340 \mathrm{Mg} / \mathrm{ha}$. As shown in Figure 4, deep forest estimated the AGB values from 0 to $340 \mathrm{Mg} / \mathrm{ha}$, and according to the AGB map, it nicely shows the regions with high and low biomass. The RF model predicted AGB values from 50 to $300 \mathrm{Mg} / \mathrm{ha}$, which shows the area with low and high biomass better than the DT model. However, RF did not perform well in areas with high biomass, which are nicely recognized in deep forest. The range of AGB estimation for DT varies from 110 to $230 \mathrm{Mg} / \mathrm{h}$. The DT did a poor job at predicting AGB for both low and high biomass regions.
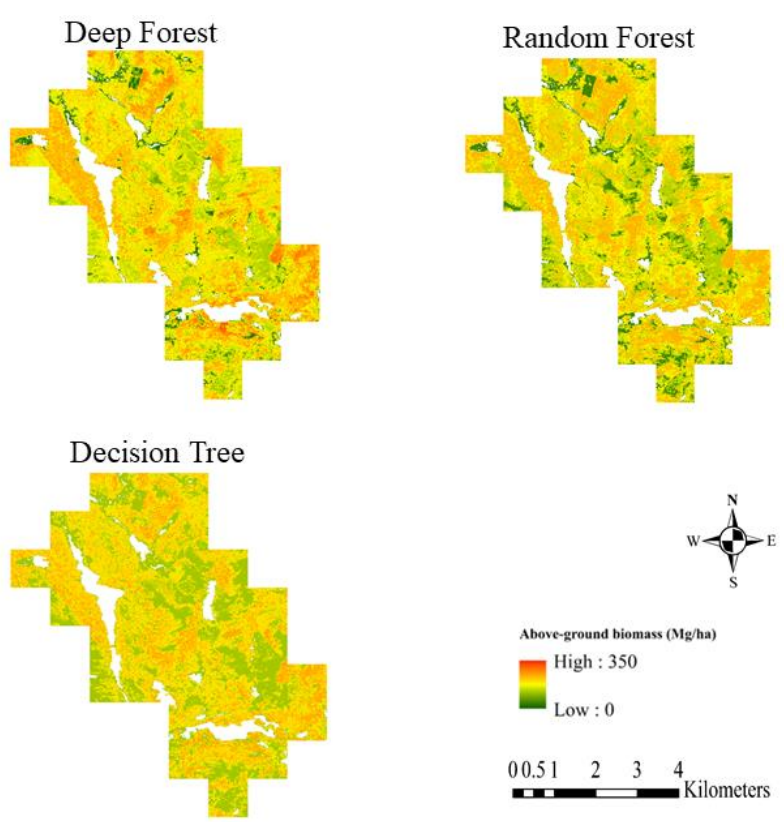

Figure 3. Above-ground biomass maps of HWF generated using decision tree-based regression models and remote sensing data. The maps are produced based on the combination of LiDAR and Landsat 5 TM data.
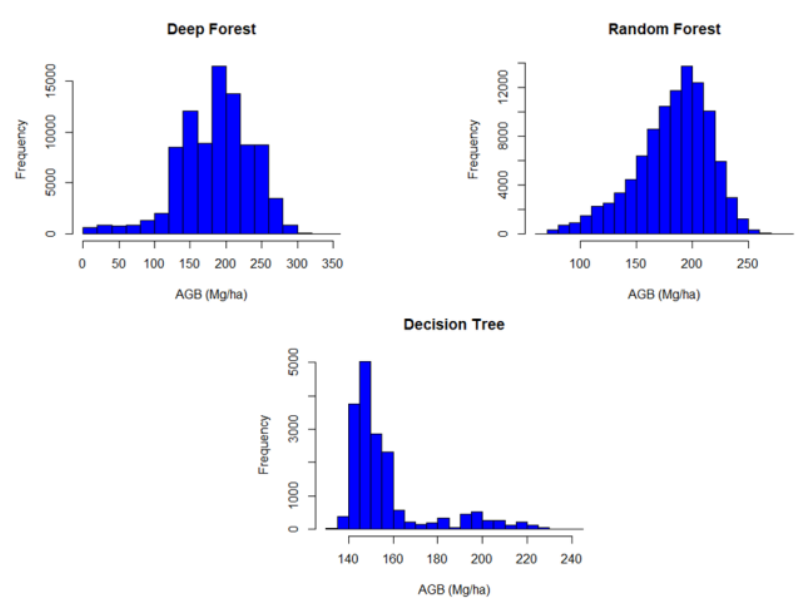

Figure 4. Histograms of AGB maps for three tree-based regression models: deep forest, RF, and DT in HWF. The AGB maps were produced using decision tree-based models and the combination of LiDAR and Landsat 5 TM imagery.

\section{CONCLUSION}

The main objective of this study was to investigate the capabilities of remote sensing data and machine learning algorithms for accurate AGB estimation. The combination of LiDAR and Landsat $5 \mathrm{TM}$ data using the deep forest regression model provided the most accurate AGB estimation. Vertical characteristics captured by LiDAR and spectral information derived by Landsat imagery could improve the AGB prediction. Deep forest, a highly competitive alternative for DNNs, outperformed RF and DT models. Thanks to the unique characteristics of deep forest, biomass can be predicted more accurately. It is recommended to use other optical and radar imagery such as Sentinel-2 and Sentinel-1 with $10 \mathrm{~m}$ spatial resolution 
and Bayesian optimization hyperparameter tuning for further studies.

\section{REFERENCES}

Ali, Jehad, Rehanullah Khan, Nasir Ahmad, and Imran Maqsood. 2012. "Random Forests and Decision Trees." International Journal of Computer Science Issues (IJCSI) 9 (5): 272.

Bastin, Jean-François, Nora Berrahmouni, Alan Grainger, Danae Maniatis, Danilo Mollicone, Rebecca Moore, Chiara Patriarca, Nicolas Picard, Ben Sparrow, and Elena Maria Abraham. 2017. "The Extent of Forest in Dryland Biomes." $\begin{array}{llll}\text { Science } & 356 & \text { (6338): } & \text { 635-38. }\end{array}$ https://doi.org/10.1126/science.aam6527.

Boudreau, Jonathan, Ross F. Nelson, Hank A. Margolis, André Beaudoin, Luc Guindon, and Daniel S. Kimes. 2008. "Regional Aboveground Forest Biomass Using Airborne and Spaceborne LiDAR in Québec." Remote Sensing of Environment 112 (10): 3876-90.

Cao, Luodan, Jianjun Pan, Ruijuan Li, Jialin Li, and Zhaofu Li. 2018. "Integrating Airborne LiDAR and Optical Data to Estimate Forest Aboveground Biomass in Arid and SemiArid Regions of China.” Remote Sensing 10 (4): 532.

Dang, An Thi Ngoc, Subrata Nandy, Ritika Srinet, Nguyen Viet Luong, Surajit Ghosh, and A. Senthil Kumar. 2019. "Forest Aboveground Biomass Estimation Using Machine Learning Regression Algorithm in Yok Don National Park, Vietnam." Ecological Informatics 50: 24-32.

Dey, Ayon. 2016. "Machine Learning Algorithms: A Review." International Journal of Computer Science and Information Technologies 7 (3): 1174-79.

Dube, Timothy, Onisimo Mutanga, Cletah Shoko, Sam Adelabu, and Tsitsi Bangira. 2016. "Remote Sensing of Aboveground Forest Biomass: A Review." Tropical Ecology 57 (May): 125-32.

Hyde, Peter, Ross Nelson, Dan Kimes, and Elissa Levine. 2007. "Exploring LiDAR-RaDAR Synergy-Predicting Aboveground Biomass in a Southwestern Ponderosa Pine Forest Using LiDAR, SAR and InSAR." Remote Sensing of Environment 106 (1): 28-38.

Issa, Salem, Basam Dahy, Taoufik Ksiksi, and Nazmi Saleous. 2020. "A Review of Terrestrial Carbon Assessment Methods Using Geo-Spatial Technologies with Emphasis on Arid Lands." Remote Sensing 12 (12): 2008.

Jenkins, Jennifer C., David C. Chojnacky, Linda S. Heath, and Richard A. Birdsey. 2003. "National-Scale Biomass Estimators for United States Tree Species." Forest Science 49 (1): 12-35.

Joshi, Neha, Edward TA Mitchard, Matthew Brolly, Johannes Schumacher, Alfredo Fernández-Landa, Vivian Kvist Johannsen, Miguel Marchamalo, and Rasmus Fensholt. 2017. "Understanding 'Saturation'of Radar Signals over Forests.” Scientific Reports 7 (1): 1-11.

Kachamba, Daud Jones, Hans Ole Ørka, Terje Gobakken, Tron Eid, and Weston Mwase. 2016. "Biomass Estimation
Using 3D Data from Unmanned Aerial Vehicle Imagery in a Tropical Woodland.” Remote Sensing 8 (11): 968.

Karlson, Martin, Madelene Ostwald, Heather Reese, Josias Sanou, Boalidioa Tankoano, and Eskil Mattsson. 2015. "Mapping Tree Canopy Cover and Aboveground Biomass in Sudano-Sahelian Woodlands Using Landsat 8 and Random Forest." Remote Sensing 7 (8): 10017-41.

Kotsiantis, Sotiris B. 2013. "Decision Trees: A Recent Overview." Artificial Intelligence Review 39 (4): 261-83.

Li, Chao, Mingyang Li, and Yingchang Li. 2020. "Improving Estimation of Forest Aboveground Biomass Using Landsat 8 Imagery by Incorporating Forest Crown Density as a Dummy Variable." Canadian Journal of Forest Research 50 (4): 390-98.

Li, Manqi, Jungho Im, and Colin Beier. 2013. "Machine Learning Approaches for Forest Classification and Change Analysis Using Multi-Temporal Landsat TM Images over Huntington Wildlife Forest." GIScience \& Remote Sensing 50 (4): 361-84.

Li, Siqi, Lindi J. Quackenbush, and Jungho Im. 2019. "Airborne Lidar Sampling Strategies to Enhance Forest Aboveground Biomass Estimation from Landsat Imagery." $\begin{array}{lllll}\text { Remote } & \text { Sensing } & 11 & \text { (16): } & 1906 .\end{array}$ https://doi.org/10.3390/rs11161906.

Li, Yingchang, Mingyang Li, Chao Li, and Zhenzhen Liu. 2020. "Forest Aboveground Biomass Estimation Using Landsat 8 and Sentinel-1A Data with Machine Learning Algorithms." Scientific Reports 10 (1): 1-12.

Mahdianpari, Masoud, Bahram Salehi, Fariba Mohammadimanesh, and Brian Brisco. 2017. "An Assessment of Simulated Compact Polarimetric SAR Data for Wetland Classification Using Random Forest Algorithm." Canadian Journal of Remote Sensing 43 (5): 468-84.

Mutanga, Onisimo, Elhadi Adam, and Moses Azong Cho. 2012. "High Density Biomass Estimation for Wetland Vegetation Using WorldView-2 Imagery and Random Forest Regression Algorithm." International Journal of Applied Earth Observation and Geoinformation 18: 399406.

Shao, Zhenfeng, and Linjing Zhang. 2016. "Estimating Forest Aboveground Biomass by Combining Optical and SAR Data: A Case Study in Genhe, Inner Mongolia, China." Sensors 16 (6): 834.

Shao, Zhenfeng, Linjing Zhang, and Lei Wang. 2017. "Stacked Sparse Autoencoder Modeling Using the Synergy of Airborne LiDAR and Satellite Optical and SAR Data to Map Forest Above-Ground Biomass." IEEE Journal of Selected Topics in Applied Earth Observations and Remote Sensing 10 (12): 5569-82.

Song, Yan-Yan, and L. U. Ying. 2015. "Decision Tree Methods: Applications for Classification and Prediction." Shanghai Archives of Psychiatry 27 (2): 130. 
Urbazaev, Mikhail, Christian Thiel, Felix Cremer, Ralph Dubayah, Mirco Migliavacca, Markus Reichstein, and Christiane Schmullius. 2018. "Estimation of Forest Aboveground Biomass and Uncertainties by Integration of Field Measurements, Airborne LiDAR, and SAR and Optical Satellite Data in Mexico." Carbon Balance and Management 13 (1): 1-20.

Zhang, Linjing, Zhenfeng Shao, Jianchen Liu, and Qimin Cheng. 2019. "Deep Learning Based Retrieval of Forest Aboveground Biomass from Combined LiDAR and Landsat 8 Data.” Remote Sensing 11 (12): 1459.

Zhao, Panpan, Dengsheng Lu, Guangxing Wang, Chuping Wu, Yujie Huang, and Shuquan Yu. 2016. "Examining Spectral Reflectance Saturation in Landsat Imagery and Corresponding Solutions to Improve Forest Aboveground Biomass Estimation." Remote Sensing 8 (6): 469

Zhou, Zhi-Hua, and Ji Feng. 2017. "Deep Forest.” ArXiv Preprint ArXiv:1702.08835. 